\title{
Modulations of NeuroD Activity Contribute to the Differential Effects of Morphine and Fentanyl on Dendritic Spine Stability
}

\author{
Hui Zheng, Yan Zeng, Ji Chu, Angel YuetFang Kam, Horace H. Loh, and Ping-Yee Law \\ Department of Pharmacology, University of Minnesota, Minneapolis, Minnesota 55455-0217
}

The cellular level of neurogenic differentiation 1 (NeuroD) is modulated differentially by $\mu$-opioid receptor agonists; fentanyl increases NeuroD level by reducing the amount of microRNA-190 (miR-190), an inhibitor of NeuroD expression, whereas morphine does not alter NeuroD level. In the current study, NeuroD activity was demonstrated to be also under agonist-dependent regulation. After $3 \mathrm{~d}$ of treatment, morphine and fentanyl decreased the activity of the $\mathrm{Ca}^{2+} /$ calmodulin-dependent protein kinase II $\alpha$ (CaMKII $\alpha$ ), which phosphorylates and activates NeuroD. Because NeuroD activity is determined by both the CaMKII $\alpha$ activity and the cellular NeuroD level, the overall NeuroD activity was reduced by morphine, but maintained during fentanyl treatment. The differential effects of agonists on NeuroD activity were further confirmed by measuring the mRNA levels of four NeuroD downstream targets: doublecortin, Notch1, neurogenic differentiation 4 , and Roundabout 1 . Decreased dendritic spine stability and $\mu$-opioid receptor signaling capability were also observed when NeuroD activity was attenuated by miR-190 overexpression or treatment with KN93, a CaMKII $\alpha$ inhibitor. The decrease could be rescued by NeuroD overexpression, which restored NeuroD activity to the basal level. Furthermore, elevating NeuroD activity attenuated the morphine-induced decrease in dendritic spine stability. Therefore, by regulating NeuroD activity, $\mu$-opioid receptor agonists modulate the stability of dendritic spines.

\section{Introduction}

Neurogenic differentiation 1 (NeuroD or BETA2) is a member of the basic helix-loop-helix transcription factor family. It is essential for the development of the CNS and the formation of the endocrine system. Severe defects in granule cells of the cerebellum and hippocampal dentate gyrus have been identified in NeuroD-null mice rescued by exogenous insulin (Cho and Tsai, 2004). The cellular level of NeuroD is differentially regulated by $\mu$-opioid receptor (OPRM1) agonists (Zheng et al., 2010b). Fentanyl suppresses the expression of microRNA-190 (miR-190), which binds complimentarily to NeuroD mRNA and decreases the mRNA and protein levels of NeuroD. Morphine treatment has no effect on the expression of miR-190 or NeuroD (Zheng et al., 2010b).

RNA interference (RNAi) against NeuroD shortens the dendrites of cerebellar granule neurons (Gaudillière et al., 2004). NeuroD functions depend on its phosphorylation on $\mathrm{Ser}^{336}$ by the $\mathrm{Ca}^{2+} /$ calmodulin-dependent protein kinase II $\alpha(\mathrm{CaMKII} \alpha)$. KN93, an inhibitor of CaMKII $\alpha$, attenuates NeuroD activity (Gaudillière et al., 2004). Thus, NeuroD activity hinges on both its expression and CaMKII $\alpha$-mediated phosphorylation. CaMKII $\alpha$

Received Dec. 8, 2009; revised March 25, 2010; accepted March 30, 2010.

The research was supported by National Institutes of Health Grants DA007339, DA016674, DA000564, and DA011806. H.H.L. and P.Y.L. are recipients of Grants K05-DA70544 and K05-DA00513, respectively. We thank Dr. Liao Dezhi for advice on preparing primary cultures and Dr. David L. Turner for NeuroD CDNA.

Correspondence should be addressed to Hui Zheng, Department of Pharmacology, University of Minnesota, 6-120 Jackson Hall, 321 Church Street SE, Minneapolis, MN 55455-0217. E-mail: zhenhui2055@hotmail.com. DOI:10.1523/JNEUROSCI.6069-09.2010

Copyright $\odot 2010$ the authors $\quad 0270-6474 / 10 / 308102-09 \$ 15.00 / 0$ is also a downstream kinase of OPRM1. Acute treatment with morphine increases the activity of CaMKII $\alpha$, whereas CaMKII $\alpha$ activity decreases after chronic morphine treatment in the rat hippocampus (Lou et al., 1999). Although how fentanyl regulates CaMKII $\alpha$ is unknown, the interaction between fentanyl and the CaMKII $\alpha$ pathway has been suggested (Chen et al., 1993).

Chronic morphine treatment decreases dendritic spine density in hippocampal neurons by causing the collapse of preexisting dendritic spines, whereas agonists that induce robust receptor internalization (e.g., etorphine and fentanyl) do not (Liao et al., $2005,2007 a, b)$. The significance of the spine collapse in the chronic drug action remains to be determined, but the observation that OPRM1 clusters on the dendritic spines (Liao et al., 2005) suggests that the collapse of spines may impair the signaling capability of OPRM1. Furthermore, the degrees of receptor internalization induced by agonists correlate with the destabilizing effects of agonists on dendritic spine stability (Liao et al., 2007b). This correlation might be the basis for the higher tolerance induced by morphine compared with fentanyl when equivalent in vivo doses of these two agonists were used (Duttaroy and Yoburn, 1995).

Although the detailed mechanisms remain unknown, a constitutively active mutant of CaMKII $\alpha$ increases dendritic spine formation (Pratt et al., 2003). In addition, CaMKII $\alpha$-mediated NeuroD phosphorylation is required for the development and maintenance of dendrite morphology (Gaudillière et al., 2004). Therefore, the CaMKII $\alpha$-NeuroD pathway may contribute to opioid-induced modulation of dendritic spine stability. In the current study, the effects of two OPRM1 agonists, morphine and 
B

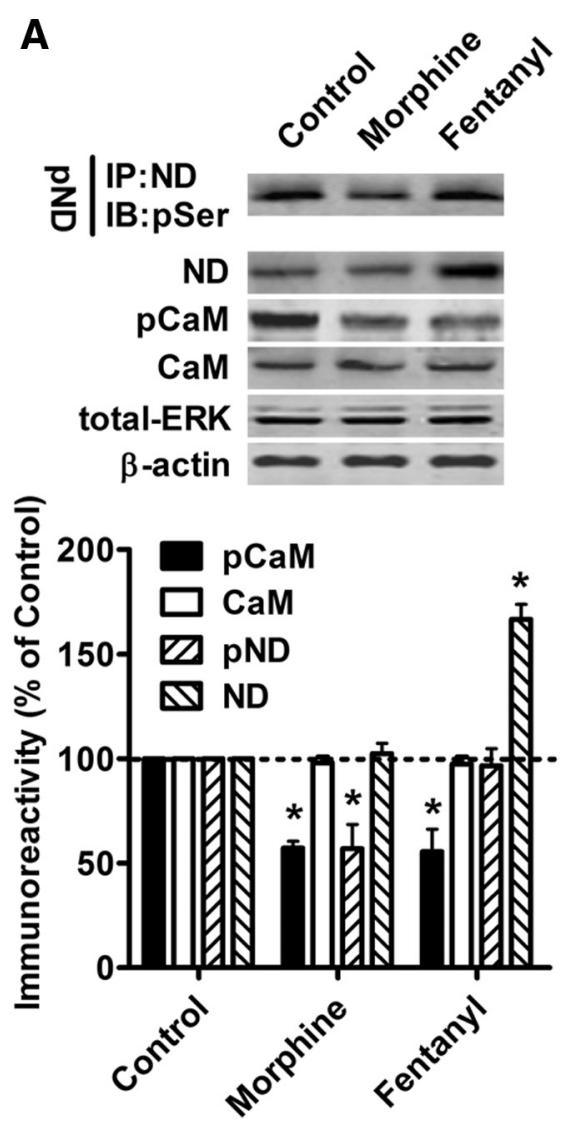

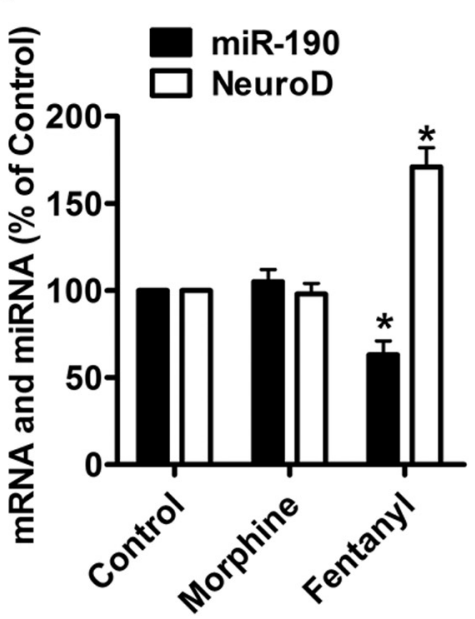

C

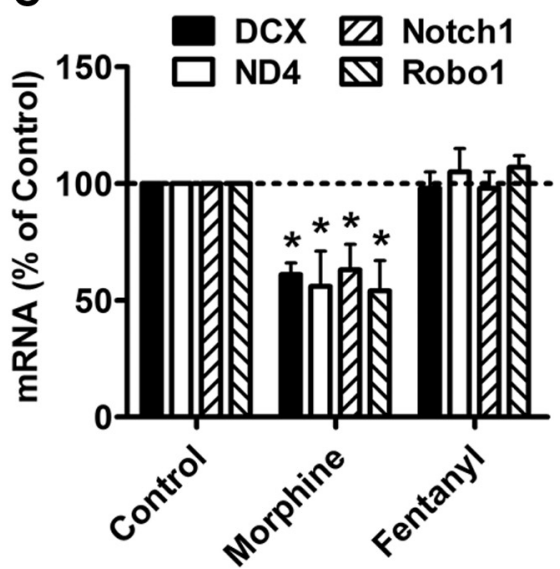

Figure 1. Morphine, but not fentanyl, decreases NeuroD activity. Primary rat hippocampal cultures were treated with PBS (control), $1 \mu \mathrm{m}$ morphine, or $10 \mathrm{~nm}$ fentanyl for $3 \mathrm{~d}$. $A$, The amount of phosphorylated serine was determined in immunoprecipitated NeuroD to indicate NeuroD phosphorylation (pND). Total NeuroD (ND) was measured in the nuclear extracts. The total CaMKII $\alpha$ (CaM), phosphorylated CaMKII $\alpha$ (pCaM), total ERK, and $\beta$-actin were detected in whole-cell lysis. Total ERK and $\beta$-actin served as internal controls. IP, Immunoprecipitation; IB, immunoblotting. $\boldsymbol{B}$, The levels of miR-190 and NeuroD mRNA were measured by real-time PCR. C, mRNA levels of DCX, ND4, Notch1, and Robo1 were determined by real-time PCR. $\boldsymbol{A}-\boldsymbol{C}$, Results were normalized against those in control samples. Error bars indicate SD. ${ }^{*} p<0.05$.

fentanyl, and CaMKII $\alpha$ on NeuroD activity were determined in primary hippocampal cultures from rats. The contribution of NeuroD activity to dendritic spine stability and to morphine-induced decrease in spine stability was also investigated.

\section{Materials and Methods}

Primary hippocampal neuron cultures and virus construction. Primary cultures of rat hippocampal neurons were prepared as described previously (Liao et al., 2007b). Matured neurons (3 weeks after plating) were used in current study. For imaging analysis, DsRed (Clontech) in pRK5 vector was transfected into neurons with Lipofectamine 2000 (Invitrogen) 1 week after plating. Treatments began on the 21 st day after plating. Livecell imaging of the neurons was captured before and after $3 \mathrm{~d}$ of treatment with a BD CARV II Confocal Imager and a Leica DMIRE2 fluorescence microscope and analyzed with IPlab 4.0 software (BD Biosciences) as previously reported (Zheng et al., 2008b). Spines were defined as dendritic protrusions with an expanded head that was $50 \%$ wider than its neck. Because there is no significant generation of new spines (Liao et al., $2007 \mathrm{~b}$ ), spine stability was measured by spine density and volume (overall DsRed fluorescence of spines). Images were adjusted to achieve comparable fluorescence intensities in the cell bodies before analysis.

Lentiviruses expressing miR-190 (190-vir: 5'-TGCTGTGATATGTTTGATATATTAGGGTTTTGGCCACTGACTGACCCTAATATCAA-

ACATATCA-3') or a control oligonucleotide (con-vir: 5' -TGCTAGAGACCAGATTACCCGGGTGTAATGGTCTCA-3') were constructed in V5-DEST using the lentiviral miR RNAi expression system (Invitrogen) according to the manufacturer's instructions. Thus, con-vir and 190-vir also express EGFP. The lentivirus expressing NeuroD (nd-vir) was generated by inserting NeuroD cDNA between the SpeI and XhoI sites of V5-DEST (Invitrogen). ECFP (with its own CMV promoter) from ECFP-N1 (Clontech) was inserted into the XbaI site of the V5-DEST. Viruses were produced by transfecting 293FT cells with V5DEST constructs together with pLP1, pLP2, and pLP-VSVG (Invitrogen). Viral titers $\left(\sim 1.2 \times 10^{6}\right.$ transducing units $\left./ \mathrm{ml}\right)$ were determined in neuroblastoma N2A cells. Infection efficiency was $>60 \%$.

Immunoprecipitation and immunoblotting. Immunoblotting and immunoprecipitation were performed as described previously (Zheng et al., 2008a). Cells were divided into three equal aliquots. One aliquot was used to prepare the nucleus extract with the NE-PER nuclear and cytoplasmic extract kit (Pierce). Nucleus extract was mixed with an antibody against NeuroD for $6 \mathrm{~h}$ and then with protein $\mathrm{G}$ beads (Invitrogen) overnight at $4^{\circ} \mathrm{C}$. Proteins bound to the beads were then extracted by using the sample buffer. NeuroD phosphorylation was determined by measuring the amounts of phosphorylated serine (pSer) in the immunoprecipitated NeuroD. The second aliquot was used to measure total NeuroD after nucleus extraction. The last aliquot was used for whole-cell lysis with the same kit to determine the levels of phosphorylated CaMKII $\alpha$, total CaMKII $\alpha$, total extracellular signalregulated kinase (ERK), and $\beta$-actin.

Total ERK and NeuroD antibodies were purchased from Cell Signaling Technology, CaMKII $\alpha$ and phosphorylated CaMKII $\alpha$ antibodies were from Genscript, and pSers from Sigma. The secondary antibody with alkaline phosphatase conjugation was from Bio-Rad Laboratory.

Real-time PCR. Total RNAs was extracted with miRNAs using the miRNeasy mini kit and reverse transcribed with miScript reverse transcription kit (both from Qiagen). Real-time PCR was done by using the miScript SYBR green PCR kit (Qiagen). GAPDH served as the internal control. Primers: NeuroD, 5'-CTTCCCGGTGCATCCCTACTCCTACC-3' and 5'-AGGAAGGGCTGGTGCAATCAGTTAGG-3'; miR-190, 5'-TGATATGTTTGATATA TTAGGT-3'; doublecortin (DCX), 5' -ACTGACATCACAGAAGCGATCAAACTGG-3' and 5'-ACTGCT AGAAGTTCCATTTGCGTCTTGG-3'; neurogenic differentiation (ND4), 5'-CAATCTGCCCTCTTGGAGAAGC GTGAGG-3' and 5'-GCGTTGAATGCACATGCCCTGAACTTACA-3'; Notch1, 5' -CACCAC GCCTCTCCCACCTGCCTGTAGC-3' and $5^{\prime}$-TGCCTGTGTGCTTAGTGTGCCCGGA GTC-3'; Roundabout 1 (Robo1), 5' -CACCAGCCAGGACATCTGCGCAGAGAGG-3' and $5^{\prime}$-GGTGGAAGG TCTCGCTTTGCTGTCTTCG-3'; GAPDH, 5' -CCTGCACCACCAACTGCTTAGC-3' and $5^{\prime}$-GCCAG TGAGCTTCCCGTTCAGC-3'.

OPRM1 signaling. The OPRM1-mediated ERK phosphorylation and adenylyl cyclase inhibition were monitored to determine signaling capability of OPRM1. Phosphorylated ERK was determined by immunoblotting as described previously (Zheng et al., 2008a). Briefly, after 5 min treatment with morphine or fentanyl, the immunoreactivity of phosphorylated ERK was measured and normalized to those of total ERK and $\beta$-actin. Adenylyl cyclase inhibition was determined by measuring cellu- 
lar cAMP level as described previously (Zhao et al., 2006). Briefly, the ability of agonist to attenuate forskolin-induced cAMP was used to indicate the decrease in adenylyl cyclase activity.

TUNEL assay. TUNEL assays were performed with ApopTag in situ apoptosis detection kit following the manufacturer's instruction. Apoptosis cells were stained with red fluorescence and counterstained with 4,6-diamidino-2phenyindole (DAPI; Santa Cruz Biotechnology). Apoptosis was quantified by calculating the percentage rate of TUNEL-positive cells in the DAPI-positive cells. As a positive control, cells were treated with $1 \mu \mathrm{M}$ staurosporin for $24 \mathrm{~h}$ to induce apoptosis.

Statistic methods. Experiments were repeated at least four times (more than eight individual neurons for image analysis). Data were analyzed and compared by one-way ANOVA with Dunnett's test as post hoc test for comparisons, except Figures $5 C$ and $7 C$ and Table 1 for which two-way ANOVA and Bonferroni's test were used.

\section{Results}

Morphine, but not fentanyl, decreases NeuroD activity

Recently we reported that chronic fentanyl, but not morphine, treatment decreased the cellular level of miR-190 in primary rat hippocampal neuron cultures and in mouse hippocampi. Because miR190 inhibited NeuroD expression, fentanyl increased the levels of NeuroD (Zheng et al., 2010b). Since NeuroD requires phosphorylation to sustain dendritic morphology, we examined whether NeuroD activity mediated the differential effects of OPRM1 agonists on dendritic spine stability.

The maximum effects of OPRM1 agonists on miR-190 expression and spine stability are observed on the third day of agonist treatment (Liao et al., 2005; Zheng et al., 2010b). In addition, 10 $\mathrm{nM}$ fentanyl induces the maximum decrease in miR-190 and $1 \mu \mathrm{M}$ morphine is equivalent to $10 \mathrm{nM}$ fentanyl in activating OPRM1 (Zheng et al., 2010b). Therefore, primary rat hippocampal neuron cultures were treated with $1 \mu \mathrm{M}$ morphine or $10 \mathrm{nM}$ fentanyl for $3 \mathrm{~d}$.

As shown in Figure 1, $A$ and $B$, fentanyl increased both the protein and mRNA levels of NeuroD, whereas morphine did not. This increase in NeuroD level was inversely related to the decrease in miR-190 expression, as previously reported (Zheng et al., 2010b). Fentanyl, but not morphine, decreased miR-190 expression (Fig. $1 B$ ).

Because phosphorylation on $\mathrm{Ser}^{336}$ of NeuroD is essential for NeuroD activity (Gaudillière et al., 2004), NeuroD activity was quantified by determining the amount of phosphorylated serine residue in immunoprecipitated NeuroD. NeuroD phosphorylation decreased after a $3 \mathrm{~d}$ morphine treatment, but was not affected by fentanyl (Fig. $1 A$ ). To confirm that the changes in NeuroD phosphorylation represented changes in NeuroD activity, the mRNA levels of four NeuroD targets (DCX, ND4, Notch1, and Robo1) were measured. NeuroD has been reported to significantly increase the mRNA levels of these four genes (Seo et al., 2007). As indicated in Figure 1C, mRNA levels of these targets decreased after morphine treatment but not after fentanyl SD. ${ }^{*} p<0.05$.
B

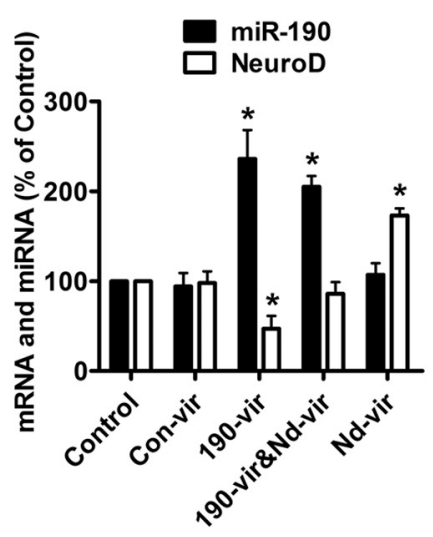

C

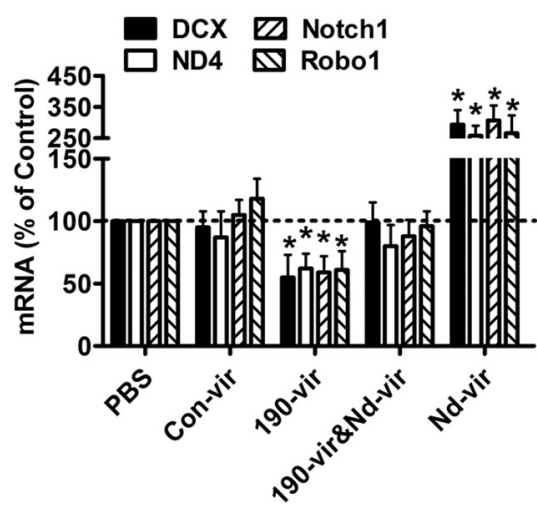

Figure 2. NeuroD level correlates with its activity. Primary cultures were treated with PBS (control), con-vir, 190-vir, 190-vir plus nd-vir, and nd-vir for $3 \mathrm{~d}$. $A$, The amounts of phosphorylated NeuroD (pND), total NeuroD (ND), total CaMKII $\alpha$ (CaM), mRNA were measured by real-time PCR C The mRNA levels of DCX ND4 , notch1, and R. $B$, The levels of miR-190 and NeuroD $A-C$, Results were normalized against those in control samples. IP, Immunoprecipitation; IB, immunoblotting. Error bars indicate

treatment, demonstrating that morphine decreased NeuroD activity, whereas fentanyl did not.

\section{Both morphine and fentanyl decrease CaMKII $\alpha$ phosphorylation}

The effects of agonists on NeuroD activity were different from those on the cellular level of NeuroD, suggesting that miR-190 was not the only factor involved in regulating NeuroD activity. Chronic morphine treatment inactivates CaMKII $\alpha$, an upstream activator of NeuroD (Lou et al., 1999; Gaudillière et al., 2004). We measured CaMKII $\alpha$ activity by determining CaMKII $\alpha$ phosphorylation at the $\mathrm{Thr}^{286}$ residue (Colbran and Brown, 2004). The level of CaMKII $\alpha$ phosphorylation decreased after chronic treatment with either morphine or fentanyl (Fig. 1A). However, the expression of CaMKII $\alpha$ was not affected. Hence, OPRM1 agonists could control NeuroD activity via two mediators, miR190 and CaMKII $\alpha$. Morphine reduced CaMKII $\alpha$ activity but not miR-190 expression, thereby decreasing NeuroD activity. In contrast, NeuroD activity was constant during fentanyl treatment, because fentanyl reduced both CaMKII $\alpha$ activity and miR-190 expression.

Modulating NeuroD cellular level controls NeuroD activity Lentiviruses were prepared to demonstrate that NeuroD activity can be modulated at the level of NeuroD protein expression. Several 190-vir and nd-vir were constructed to ectopically express miR-190 and NeuroD, while con-vir was constructed to 
A
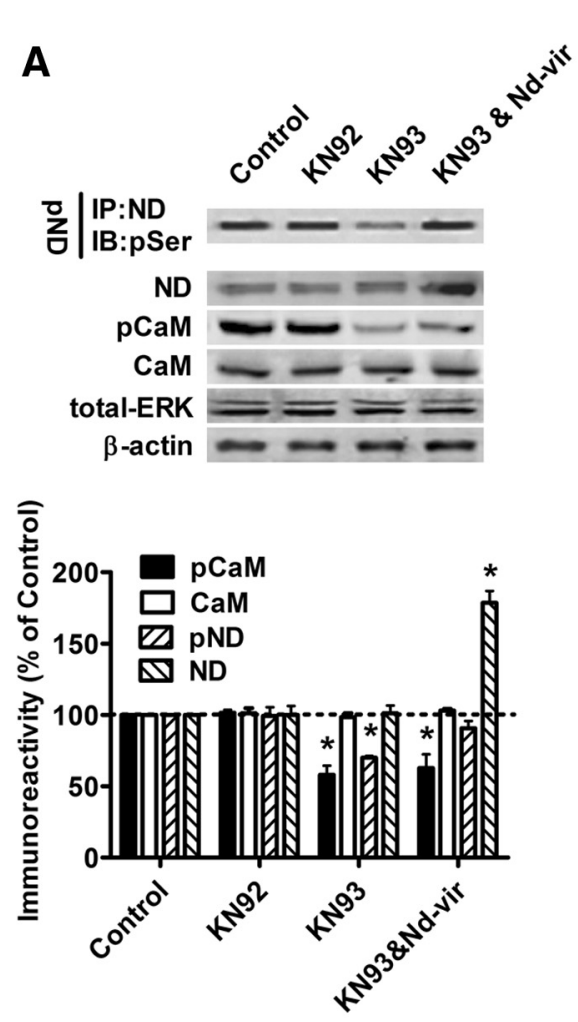

B

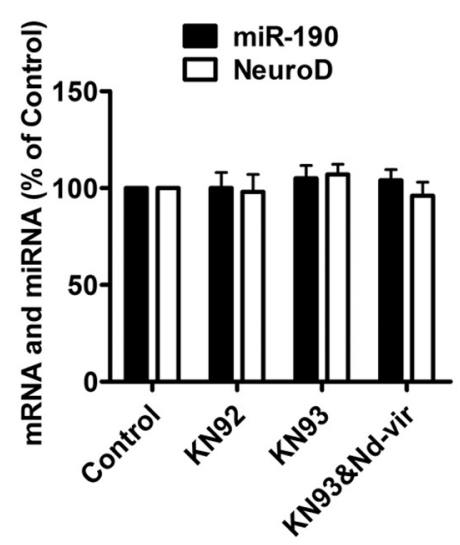

C

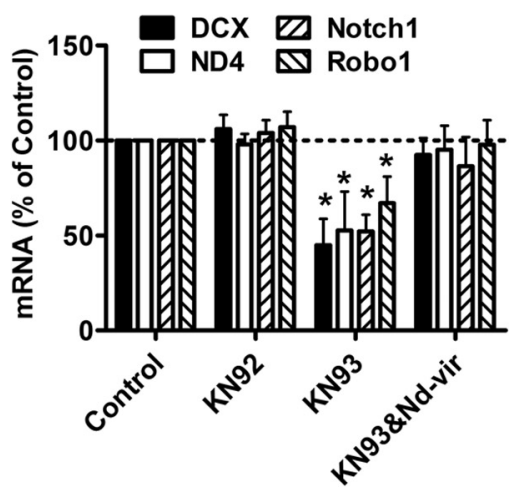

Figure 3. NeuroD activity correlates with its phosphorylation by CaMKII $\alpha$. Primary cultures were treated with PBS (control), KN92 $(2 \mu \mathrm{M})$, KN93 $(2 \mu \mathrm{M})$, or KN93 (2 $\mu \mathrm{M})$ plus nd-vir for 3 d. $A$, The amount of phosphorylated NeuroD (pND), total NeuroD (ND), total CaMKII $\alpha(\mathrm{CaM})$, phosphorylated CaMKII $\alpha$ (pCaM), total ERK, and $\beta$-actin were determined as in Figure $1 A$. $\boldsymbol{B}$, The levels of miR-190 and NeuroD mRNA were measured by real-time PCR. C, The mRNA levels of DCX, ND4, Notch1, and Robo1 were determined with real-time PCR. $\boldsymbol{A}-\boldsymbol{C}$, Results were normalized against those in control samples. IP, Immunoprecipitation; IB, immunoblotting. Error bars indicate SD. ${ }^{*} p<0.05$.
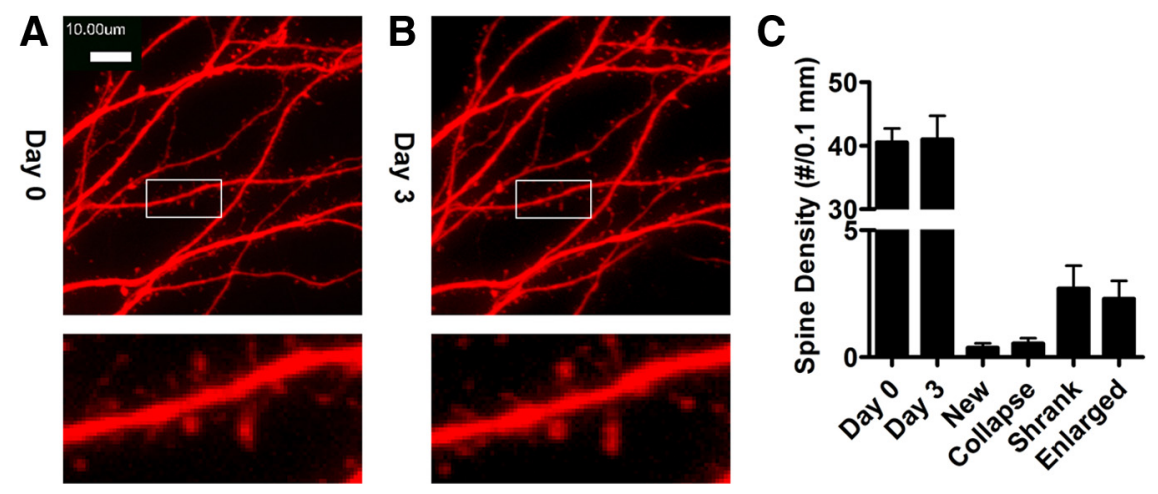

Figure 4. Spine collapse and new spine formation during control PBS treatment. Primary cultures from rat hippocampal neurons were treated with PBS (control) for 3 d. $\boldsymbol{A}, \boldsymbol{B}$, Live-cell images on day $0(\boldsymbol{A})$ and day $3(\boldsymbol{B})$ of a representative neuron were presented. Spine densities on day 0 and day 3 , the numbers of newly formed spines, and the number of spines that collapsed, shrank, or enlarged during $3 \mathrm{~d}$ are also summarized in C. Error bars indicate SD.

serve as a negative control. As indicated in the Figure 2, $A$ and $B$, con-vir did not change the cellular NeuroD level (neither protein nor mRNA levels) or miR-190 level. However, a significant increase in miR-190 level was observed $3 \mathrm{~d}$ after 190-vir infection. Decreased NeuroD expression was observed at the same time, which is consistent with the inhibitory effects of miR-190 on NeuroD expression. In parallel, infection with nd-vir did not affect miR-190, but increased NeuroD at both the mRNA and protein levels (Fig. $2 A, B$ ). When primary hippocampal neuron cultures were infected with 190-vir and ndvir simultaneously, no measurable decrease in NeuroD expression was observed, even though miR-190 was elevated (Fig. 2A,B). Thus, the inhibitory effects of miR-190 on NeuroD level can be reversed by nd-virmediated overexpression of NeuroD.

Changes in NeuroD phosphorylation were also monitored in primary hippocampal neuron cultures infected with these viruses. 190-vir decreased and nd-vir increased the amount of phosphorylated NeuroD (Fig. 2A). NeuroD phosphorylation was maintained at the basal level when primary hippocampal neuron cultures were infected with both 190-vir and nd-vir (Fig. 2A). Consistently, 190-vir decreased and nd-vir increased the mRNA levels of DCX, ND4, Notch1, and Robo1 (Fig. 2C). When 190-vir and nd-vir were used to coinfect primary cultures, mRNAs levels of these four targets were close to the basal levels (Fig. 2C), as was NeuroD phosphorylation. These viruses were then used to modulate NeuroD levels, thereby controlling NeuroD activity.

\section{Modulating CaMKII $\alpha$ activity also controls NeuroD activity}

KN92 and KN93 were used to demonstrate that NeuroD activity can be modulated at the level of NeuroD phosphorylation. KN93 is a specific inhibitor for CaMKII, whereas KN92 is a structural analog of KN93 that does not inhibit CaMKII (Sumi et al., 1991). The activity of CaMKII $\alpha$ was indicated by its autophosphorylation on $\mathrm{Thr}^{286}$ (Wayman et al., 2008), and KN93 has been reported to decrease the autophosphorylation of CaMKII $\alpha$ (Sumi et al., 1991). As predicted, no significant change in CaMKII $\alpha$ phosphorylation or CaMKII $\alpha$ expression was observed after $3 \mathrm{~d}$ of treatment with $2 \mu \mathrm{M}$ KN92 (Fig. $3 A$ ). However, $2 \mu \mathrm{M}$ KN93 decreased Thr ${ }^{286}$ phosphorylation of CaMKII $\alpha$ but left CaMKII $\alpha$ expression unchanged (Fig. $3 A)$. The two inhibitors had no effects on NeuroD and miR-190 levels (Fig. $3 A, B)$. However, KN93, but not KN92, decreased NeuroD activity as indicated by NeuroD phosphorylation and the mRNA levels of four NeuroD targets (Fig. $3 A, C)$. Thus, NeuroD activity can be altered by modulating CaMKII $\alpha$ activity without changes in NeuroD expression.

NeuroD overexpression, however, was able to reverse the KN93-induced decrease in NeuroD activity. In the primary hippocampal neuron cultures infected with nd-vir and treated with KN93, decrease in CaMKII $\alpha$ phosphorylation was observed (Fig. $3 A$ ). However, due to the nd-vir-mediated increase in NeuroD, the amount of NeuroD being phosphorylated and mRNA levels 
of the four NeuroD targets in these primary neuron cultures were not significantly different from those observed in control primary neuron cultures (Fig. $3 A, C)$. Therefore, NeuroD activity can be maintained even when CaMKII $\alpha$ is inhibited.

\section{NeuroD activity is involved in} maintaining dendritic spine stability Before examining the relationship between NeuroD activity and dendritic spine stability, we monitored dendritic morphology and spine density of the primary hippocampal neuron cultures under control conditions. DsRed in pRK5 vector was transfected into primary rat hippocampal neuron cultures 1 week after plating. Two weeks after transfection, dendritic spines were monitored for $3 \mathrm{~d}$. As shown in Figure 4, $A$ and $B$, the formation of new spines $(0.4 \pm 0.2$ spine $/ 0.1$ $\mathrm{mm}, n=8)$ and the collapse of existing spines ( $0.5 \pm 0.2$ spine $/ 0.1 \mathrm{~mm}$ dendrite, $n=8$ ) were not significant during a $3 \mathrm{~d}$ treatment with PBS. The density of dendritic spine was determined to be $41 \pm 2$ spine/ $0.1 \mathrm{~mm}$ dendrite $(n=8)$ and $41 \pm 4$ spine/0.1 $\mathrm{mm}$ dendrite $(n=8)$ on day 0 and day 3 , respectively (Fig. 4C). Overall volume of dendritic spines was measured by determining DsRed fluorescence intensity. Overall volume of dendritic spines was constant during the $3 \mathrm{~d}$, and the spine volume on day 3 was $102 \pm 5 \%$ of that on day 0 . In addition, the spines were stable during the $3 \mathrm{~d}$ treatment. Only $5 \%$ spines (2.7 \pm 0.9 spine $/ 0.1 \mathrm{~mm}$ dendrite, $n=8$ ) shrank by $>20 \%$, and only $6 \%$ spines (2.3 \pm 0.7 spine $/ 0.1 \mathrm{~mm}$ dendrite, $n=8$ ) enlarged by $>20 \%$.

The effects of various treatments on dendritic spine stability were then examined. As shown in Figure 5A, successful infection of primary cultures with con-vir or 190-vir was represented by the fluorescence of EGFP, while the fluorescence of ECFP represented the successful infection by nd-vir. In 190-vir-infected primary neuron cultures, spine density decreased from $43 \pm 3.2$ spine $/ 0.1 \mathrm{~mm}(n=$ 9) on day 0 to $33 \pm 3.1$ spine $/ 0.1 \mathrm{~mm}(n=9)$ on day 3 (Fig. $5 B$ ). A $55 \pm 17 \%(n=9)$ decrease in spine volume was also observed (Fig. 5 C). This loss in spine density and in spine volume suggests a decrease in spine stability, because few new spines formed in control cultures during the $3 \mathrm{~d}$ treatment (Fig. $4 C$ ). The decrease in spine volume appeared larger than that in spine density because some spines only shrank but did not collapse. Similar decrease in spine stability was observed in primary cultures treated with KN93, but not in those treated with con-vir or KN92 (Fig. $5 A-C)$. In addition, spine collapse was not due to the apoptosis of primary neurons, because apoptosis rates in treated primary neuron cultures were not higher than that in the control cultures (Fig. 5D).

Since NeuroD activity was decreased by the treatment with 190-vir or KN93 (Figs. 2 and 3), there is a correlation between decreased NeuroD activity and reduced spine stability. To further test this correlation, nd-vir was used to infect primary cultures together with 190-vir or KN93. As shown in Figure $5 A-C$, the spine collapse induced by 190-vir and KN93 was restored by nd-vir. However, nd-vir alone did not change spine density or spine volume (Fig. $5 A-C$ ), suggesting that the basal NeuroD activity is not limiting for spine formation and that the recovery of spine density/volume is not due to the ability of nd-vir to increase spine density/volume, but due to the ability of nd-vir to restore NeuroD activity.

NeuroD activity mediates the effects of agonists on spine stability

As noted above, morphine decreased, whereas fentanyl maintained, NeuroD activity (Fig. 1). Meanwhile, morphine has been reported to reduce spine stability, but fentanyl did not affect spine stability (Liao et al., 2005, 2007a). Thus, we examined whether morphine decreases spine stability by suppressing NeuroD activity. Although $1 \mathrm{~d}$ of morphine treatment significantly decreased spine stability, the effect of $3 \mathrm{~d}$ of morphine treatment 

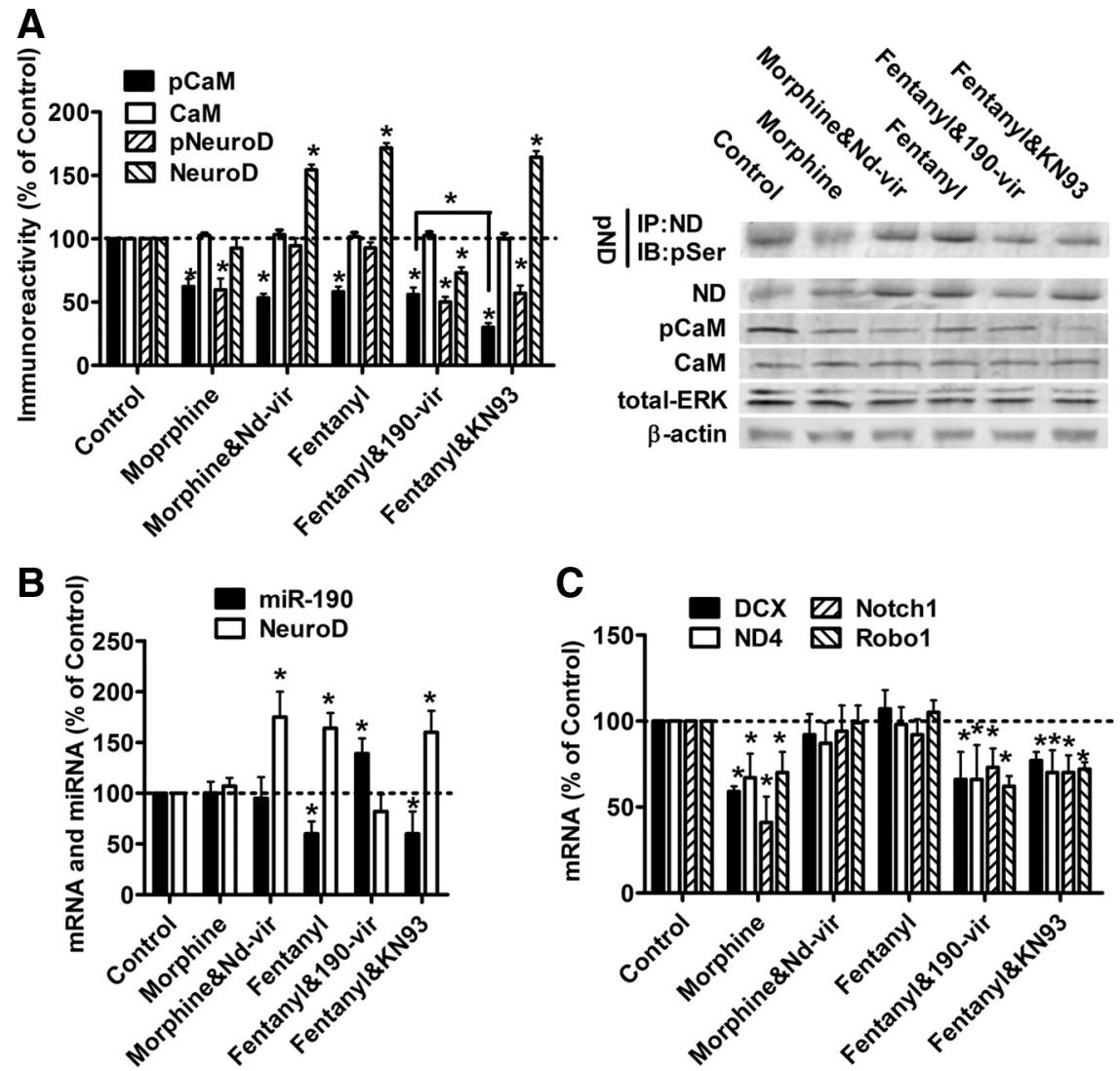

Figure 6. Overexpressing NeuroD counteracts morphine-induced decrease in NeuroD activity. Primary cultures were treated with PBS, morphine (1 $\mu \mathrm{M})$, morphine (1 $\mu \mathrm{M})$ plus nd-vir, fentanyl (10 nM), fentanyl (10 nM) plus 190-vir, or fentanyl (10 nM) plus KN93 for 3 d. $\boldsymbol{A}$, The amount of phosphorylated NeuroD (pNeuroD), total NeuroD, total CaMKIl $\alpha$ (CaM), phosphorylated CaMKIl $\alpha$ (pCaM), total ERK, and $\beta$-actin were determined as in Figure $1 A$. B, The levels of miR-190 and NeuroD (ND) mRNA were measured by real-time PCR. IP, Immunoprecipitation; IB, immunoblotting. $C$, The mRNA levels of DCX, ND4, Notch1, and Rob01 were determined by real-time PCR. $\boldsymbol{A}-\boldsymbol{C}$, Results were normalized against those in control samples. Error bars indicate $S D .{ }^{*} p<0.05$.

spine stability was also impaired (Fig. 7A). Spine densities were $41 \pm 3.9$ spine/0.1 $\mathrm{mm}(n=12)$ and $39 \pm 4.7$ spine $/ 0.1 \mathrm{~mm}$ $(n=12)$ on day 0 and day 3 , respectively (Fig. $7 B$ ). In addition, spine volume on day 3 was $87 \pm 12 \%$ of that on day 0 (Fig. $7 C)$. Since, restoring NeuroD activity can attenuate the inhibitory effect of morphine on spine stability, this suggests that morphine decreases spine stability by suppressing NeuroD activity.

Fentanyl regulated NeuroD activity via two mechanisms: it reduced NeuroD activation by inhibiting CaMKII $\alpha$ activity, but it also enhanced NeuroD expression by suppressing miR-190 (Fig. 6). The net result is that fentanyl did not alter the levels of phosphorylated NeuroD and the mRNA of the four NeuroD targets (Fig. $6 A, C)$. In fentanyl-treated primary cultures, spine densities were $40 \pm 4.0$ spine/ $0.1 \mathrm{~mm}(n=12)$ and $40 \pm 6$ spine $/ 0.1 \mathrm{~mm}$ $(n=12)$ on day 0 and day 3 , respectively (Fig. $7 B$ ). In addition, spine volume on day 3 was $92 \pm 8 \%$ of that on day 0 (Fig. 7C). Therefore, fentanyl neither decreased NeuroD activity nor reduced spine stability.

If the NeuroD activity mediated the effects of OPRM1 agonists on spine stability, decreasing NeuroD activity during fentanyl treatment should enable fentanyl to induce spine collapse. Thus, 190-vir and KN93 were used along with fentanyl to further explore the relationship between NeuroD activity and dendritic spine stability. When primary hippocam-

was more pronounced, so we monitored the morphology of primary cultures for $3 \mathrm{~d}$ under opioid treatment (Liao et al., 2005). To determine whether decreased NeuroD activity contributed to the destabilizing effect of morphine on dendritic spines, 190vir and nd-vir were used simultaneously with OPRM1 agonists to modulate the NeuroD activity.

Consistent with Figure 1 and a previous report (Zheng et al., 2010b), $3 \mathrm{~d}$ of morphine treatment decreased CaMKII $\alpha$ phosphorylation and NeuroD activity (both the amount of phosphorylated NeuroD and the mRNA levels of NeuroD targets), but did not alter the expression of miR-190 or NeuroD (Fig. 6). In addition, $3 \mathrm{~d}$ of morphine treatment reduced spine stability (Fig. $7 A)$. Spine densities were $43 \pm 3.3$ spine/0.1 $\mathrm{mm}(n=12)$ and $33 \pm 5.1$ spine/ $0.1 \mathrm{~mm}(n=12)$ on day 0 and day 3 , respectively (Fig. $7 B$ ), and spine volume decreased by $44 \pm 18 \%$ after $3 \mathrm{~d}$ of morphine treatment (Fig. $7 C$ ). Thus, chronic morphine treatment decreased NeuroD activity and spine stability.

If morphine decreased spine stability by suppressing NeuroD activity, restoring NeuroD activity should prevent morphine-induced spine collapse. When nd-vir was used to infect primary neurons simultaneously with morphine treatment, the cellular level of NeuroD increased significantly (Fig. 6A). However, NeuroD phosphorylation level and mRNA levels of the four NeuroD targets were close to the basal levels (Fig. 6A, C), suggesting that the morphine-induced decrease in NeuroD activity was largely reversed. Moreover, the ability of morphine to reduce pal neuron cultures were infected with 190-vir at the initiation of $3 \mathrm{~d}$ of fentanyl treatment, fentanyl-induced increase in NeuroD cellular level was blocked (Fig. 6A,B). In addition, phosphorylated NeuroD (Fig. 6A) and mRNAs of the four NeuroD targets were reduced. In fentanyl and 190-vir-treated primary cultures, spine densities were $41 \pm 3.5$ spine $/ 0.1 \mathrm{~mm}(n=12)$ and $31 \pm 5.5$ spine $/ 0.1 \mathrm{~mm}(n=12)$ on day 0 and day 3 , respectively (Fig. $7 B$ ), and spine volume on day 3 was $56 \pm 12 \%$ of that on day 0 (Fig. 7C).

CaMKII $\alpha$ activity in primary cultures treated with both fentanyl and KN93 was lower than that in primary cultures treated with fentanyl alone (Fig. 6A), although KN93 did not alter miR190 or NeuroD cellular level. The inclusion of this CaMKII $\alpha$ inhibitor during fentanyl treatment reduced the amount of phosphorylated NeuroD and mRNA levels of the four NeuroD targets (Fig. 6C). In primary cultures treated with both fentanyl and KN93, spine densities were $40 \pm 3.3$ spine/ $0.1 \mathrm{~mm}(n=12)$ and $29 \pm 4.3$ spine $/ 0.1 \mathrm{~mm}(n=12)$ on day 0 and day 3 , respectively (Fig. $7 B$ ), and spine volume on day 3 was $66 \pm 9 \%$ of that on day 0 (Fig. 7C). Therefore, decreasing NeuroD activity during fentanyl treatment leads to spine collapse.

Both KN92 and KN93 were dissolved in DMSO. However, the observations with KN93 were not due to vehicle effects, because KN92, which is also dissolved in DMSO, did not affect the NeuroD phosphorylation (Fig. 3) or dendritic spine stability (Fig. 5). To assure there is no influence from DMSO, primary cultures 
were treated with DMSO $(0.1 \% \mathrm{v} / \mathrm{v}$, because KN93 and KN92 used as $1000 \times$ ) for $3 \mathrm{~d}$. As indicated in Figure 7, DMSO did not induce significant change in dendritic spine stability or apoptosis. Thus, the decrease in spine stability was due to the KN93 inhibition on CaMKII $\alpha$ activity.

Spine stability is required for OPRM1 signaling

Chronic morphine, but not fentanyl, induces spine collapse (Liao et al., 2005). Morphine also has a higher ability than fentanyl to induce tolerance (Duttaroy and Yoburn, 1995). Thus, we examined whether morphine-induced spine collapse contributes to OPRM1 signaling. To minimize the influence of receptor desensitization during agonist treatment, miR190 and nd-vir were used to mimic the effects of morphine and fentanyl on spine stability.

190-vir and nd-vir were first used to infect primary neurons for $3 \mathrm{~d}$ to alter NeuroD activity and dendritic spine stability. Morphine or fentanyl was then added to the cells to determine the signaling capability of OPRM1 by measuring ERK phosphorylation and adenylyl cyclase inhibition (Table 1). Morphine and fentanyl increased ERK phosphorylation to $175 \pm 15 \%$ and $178 \pm 8 \%$, respectively, of the basal level in control primary cultures. The maximum inhibition of adenylyl cyclase induced by morphine and fentanyl was $30 \pm 2.7 \%$ and $29 \pm 5.1 \%$, respectively. When con-vir was used to infect the primary cultures, no significant difference was observed in the signaling capability of OPRM1 (Table 1). However, if NeuroD activity and spine stability were reduced by 190 -vir infection, the signaling capability of OPRM1 decreased significantly. Morphine and fentanyl only induced ERK phosphorylation to $125 \pm$ $8 \%$ and $128 \pm 4 \%$ of basal level, respectively. The maximum inhibition of adenylyl cyclase was also attenuated by morphine $(10 \pm 3.1 \%)$ and fentanyl $(7.7 \pm 2.8 \%)$. The decreased signaling capability of OPRM1 can be rescued by simultaneously infecting the primary cultures with nd-vir and 190-vir. In these primary cultures, not only the NeuroD activity and spine stability, but also the OPRM1 signaling capability, were close to the control level (Table 1). Therefore, NeuroD activity or spine stability is required for the normal function of OPRM1. By decreasing the activity of NeuroD, chronic morphine treatment may decrease the signaling capability of OPRM1.

\section{Discussion}

This current study clearly indicates that these two agonists can induce different overall cellular responses by activating the same signaling molecules. As summarized in Figure 8, both fentanyl and morphine decrease NeuroD phosphorylation by inhibiting CaMKII $\alpha$ activity. However, fentanyl, but not morphine, increases the cellular NeuroD content by decreasing miR-190 level. These two effects cross talk with each other and result in the different modulations on NeuroD activity. Therefore, morphine decreases NeuroD activity, whereas fentanyl maintains NeuroD activity. Since NeuroD activity appears to be required for the maintenance of dendritic spine stability by decreasing NeuroD activity, morphine reduces spine stability and fentanyl does not.

To achieve maximum effect, the concentrations $(1 \mu \mathrm{M}$ morphine and $10 \mathrm{~nm}$ fentanyl) and treatment time ( $3 \mathrm{~d})$ used in the current study were determined based on previous reports (Liao et al., 2005; Zheng et al., 2010b). Therefore, although only one concentration for each agonist and one treatment time were tested, current observations still reflect the effects of OPRM1 agonists on NeuroD activity and spine stability.

Both miR-190 and CaMKII $\alpha$ have other targets in addition to NeuroD. However, their abilities to influence dendritic spine stability are primarily mediated by NeuroD, since overexpressing NeuroD in primary hippocampal neuron cultures by nd-vir infection attenuated the inhibitory effects of miR-190 and KN93 on spine stability (Figs. 2-4). These abilities of CaMKII $\alpha$ and NeuroD to regulate spine stability correlate with their reported functions. CaMKII $\alpha$ is essential for synaptic transmission and dendritic morphology (Colbran and Brown, 2004), and its phosphorylation is associated with microtubule stabilization and dendrite formation (Vaillant et al., 2002). NeuroD is also implicated in the control of dendritic morphology (Gaudillière et al., 2004), and it regulates a vast number of genes, some of which play critical roles in the CNS (Seo et al., 2007). Although the mechanism of how NeuroD activity contributes to the mainte- 
Table 1. NeuroD activity and spine stability influence OPRM1 signaling

\begin{tabular}{|c|c|c|c|c|c|c|}
\hline & \multicolumn{3}{|l|}{ Morphine } & \multicolumn{3}{|l|}{ Fentanyl } \\
\hline & \multicolumn{2}{|l|}{ AC inhibition } & \multirow{2}{*}{$\begin{array}{l}\text { Phosphorylated } \\
\text { ERK (\% of control) }\end{array}$} & \multicolumn{2}{|l|}{ AC inhibition } & \multirow{2}{*}{$\begin{array}{l}\text { Phosphorylated } \\
\text { ERK (\% of control) }\end{array}$} \\
\hline & $K_{1}(\mathrm{~nm})$ & Maximum inhibition (\%) & & $K_{1}(\mathrm{pm})$ & Maximum inhibition (\%) & \\
\hline PBS & $24.2 \pm 6.5$ & $30.0 \pm 2.7$ & $173 \pm 15$ & $165 \pm 40$ & $29.2 \pm 5.1$ & $178 \pm 8$ \\
\hline Con-vir & $26.0 \pm 3.5$ & $28.9 \pm 4.2$ & $165 \pm 16$ & $201 \pm 45$ & $27.4 \pm 4.3$ & $174 \pm 10$ \\
\hline 190-vir & $67.6 \pm 8.1^{*}$ & $10.0 \pm 3.1^{*}$ & $125 \pm 8^{*}$ & $857 \pm 109^{*}$ & $7.7 \pm 2.8^{*}$ & $128 \pm 4^{*}$ \\
\hline 190-vir plus nd-vir & $38.7 \pm 9.4$ & $21.6 \pm 3.2$ & $151 \pm 5$ & $256 \pm 48$ & $23.1 \pm 3.8$ & $159 \pm 13$ \\
\hline nd-vir & $22.2 \pm 4.8$ & $33.4 \pm 2.5$ & $177 \pm 25$ & $178 \pm 27$ & $27.2 \pm 3.1$ & $176 \pm 13$ \\
\hline
\end{tabular}

Rat primary hippocampal neuron cultures were infected with PBS, con-vir, 190-vir, 190-vir plus nd-vir, or nd-vir for $3 \mathrm{~d}$. Cells were then treated for 5 min with $1 \mu \mathrm{m}$ morphine or $10 \mathrm{~nm}$ fentanyl before ERK phosphorylation was measured. Serial doses of morphine $(10 \mu \mathrm{M} \sim 0.01 \mathrm{~nm})$ or etorphine $(100 \mathrm{nM} \sim 0.1 \mathrm{pM})$ were used to determine the dose response of the agonists to induce adenylyl cyclase (AC) inhibition as described in Materials and Methods. To determine ERK phosphorylation, the immunoreactivity of phosphorylated ERK was normalized to the immunoreactivity of total ERK and $\beta$-actin, and the results were further normalized against those in untreated cells. Adenylyl cyclase inhibition induced by agonists was indicated by both IC50 $\left(K_{1}\right)$ and the maximum inhibition. No difference in the basal levels of OPRM1 signaling was observed in different groups.

*Decreased signaling capability of OPRM1.

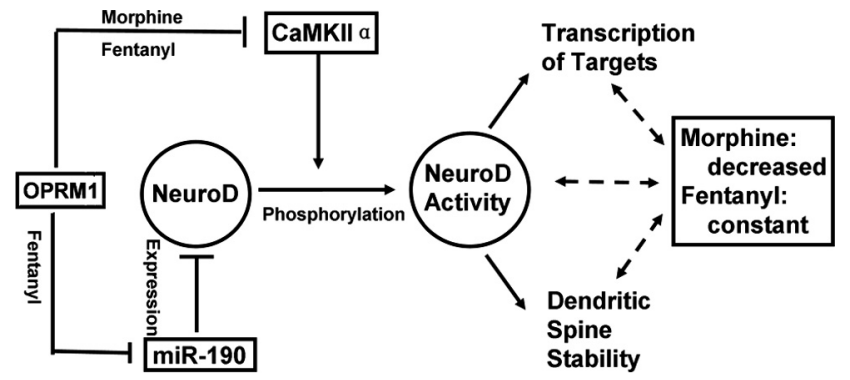

Figure 8. Schematic summary of agonist-dependent regulation on NeuroD activity.

nance of spine stability requires further investigation, the ability of NeuroD to function as transcription activator is clearly critical. Two of the four NeuroD targets investigated in the current studies, DCX and Notch1, may control adult neurogenesis. DCX is an important transcription factor involved in the migration and differentiation processes during adult neurogenesis (von Bohlen und Halbach, 2007). Notch1 signaling is critical for dendrite morphology of newborn neurons in the postnatal dentate gyrus (Breunig et al., 2007). Since formation of functional dendritic spine contributes to adult neurogenesis, NeuroD may regulate dendritic spine stability via these two factors. NeuroD also controls the transcription of postsynaptic EPH receptor B1 and B2 (Seo et al., 2007), whose interactions with presynaptic ephrins are critical for the formation and stabilization of dendritic spines (Kayser et al., 2008). In addition, NeuroD controls the formation of the endocrine system (Chae et al., 2004), which is another possible route for NeuroD to affect spine stability.

Fentanyl decreases the level of miR-190, which depends on $\beta$-arrestin-mediated ERK phosphorylation and nucleus translocation of the phosphorylated ERK. Because morphine uses the PKC pathway for ERK phosphorylation and retains phosphorylated ERK in the cytosol, it does not regulate miR-190 level (Zheng et al., 2008a,b, 2010b). This current study provides more information on agonist-dependent signaling. Both morphine and fentanyl decrease CaMKII $\alpha$ activity, but they regulate NeuroD activity differentially (Fig. 8). This interaction among multiple signaling events constitutes a complex network of agonistdependent signaling. These observations further support our hypothesis that agonist-dependent regulation of gene expression (e.g., miR-190) links agonists-dependent signaling (e.g., ERK phosphorylation) to the agonist-dependent response (e.g., changes in NeuroD activity and spine stability) (Zheng et al., 2008a, 2010a).

The different abilities of agonists to influence spine stability have been attributed to the different levels of receptor internal- ization induced by agonists, because blocking OPRM1 internalization with a dominant-negative Dynamin I (K44E) enables fentanyl to decrease the stability of dendritic spines (Lin et al., 2009). How OPRM1 internalization contributes to spine stability is uncertain, but this study provides a probable explanation. Blocking receptor internalization with $\mathrm{K} 44 \mathrm{E}$ maintains $\beta$-arrestin on the cell-surface membrane (Gáborik et al., 2001; Schafer, 2004). Fentanyl-induced decreases in miR-190 level are dependent on $\beta$-arrestin-mediated ERK phosphorylation and the nucleus translocation of phosphorylated ERK (Zheng et al., 2008a, $2010 \mathrm{~b})$. Because of the ability of $\beta$-arrestin to scaffold ERK, to translocate into nucleus, and to activate transcription (Ma and Pei, 2007; Violin and Lefkowitz, 2007), $\beta$-arrestin retained at the cell-surface membrane could impair the ability of fentanyl to decrease miR-190 expression. Consequently, without the observed increase in the NeuroD level, CaMKII $\alpha$ inhibition by fentanyl will decrease NeuroD activity. Hence, when receptor internalization is blocked, fentanyl may function like morphine to decrease NeuroD activity and subsequent spine stability.

Morphine, but not fentanyl, decreased NeuroD activity and spine stability. If the decreased NeuroD activity and spine stability was mimicked by 190 -vir infection, a significant decrease in OPRM1 signaling capability was observed (Table 1). These data imply that chronic morphine, but not fentanyl, treatment can decrease the signaling capability or responsibility of OPRM1, which may explain why morphine induces higher tolerance than does fentanyl.

We have demonstrated the abilities of OPRM1 agonists to regulate NeuroD activity and subsequent dendritic spine stability. Morphine treatment delays the growth of cortical dendrites (Ricalde and Hammer, 1990; Mei et al., 2009). Morphine injection decreases the dendritic spine density in the hippocampus and nucleus accumbens (Robinson et al., 2002; Diana et al., 2006). Although the effects of other opioids on spine stability have not been reported in vivo, it is still reasonable to suggest that the signaling cascade identified in the current study also has similar functions in vivo and may contribute to regulatory effects of opioids on dendrite morphology. Since NeuroD plays critical roles in regulating adult neurogenesis (von Bohlen und Halbach, 2007), OPRM1-mediated regulation of NeuroD activity may also contribute to the adult neurogenesis. Chronic morphine has been reported to decrease the rate of adult neurogenesis in dentate gyrus (Eisch et al., 2000). In addition, the hippocampus is highly related to learning and memory processes. By targeting NeuroD activity and spine stability, OPRM1 agonists may regulate the functions of the hippocampus in learning and memory that have been reported previously (Pu et al., 2002; Bao et al., 2007). 


\section{References}

Bao G, Kang L, Li H, Li Y, Pu L, Xia P, Ma L, Pei G (2007) Morphine and heroin differentially modulate in vivo hippocampal LTP in opiatedependent rat. Neuropsychopharmacology 32:1738-1749.

Breunig JJ, Silbereis J, Vaccarino FM, Sestan N, Rakic P (2007) Notch regulates cell fate and dendrite morphology of newborn neurons in the postnatal dentate gyrus. Proc Natl Acad Sci U S A 104:20558-20563.

Chae JH, Stein GH, Lee JE (2004) NeuroD: the predicted and the surprising. Mol Cells 18:271-288.

Chen Y, Mestek A, Liu J, Yu L (1993) Molecular cloning of a rat kappa opioid receptor reveals sequence similarities to the mu and delta opioid receptors. Biochem J 295:625-628.

Cho JH, Tsai MJ (2004) The role of BETA2/NeuroD1 in the development of the nervous system. Mol Neurobiol 30:35-47.

Colbran RJ, Brown AM (2004) Calcium/calmodulin-dependent protein kinase II and synaptic plasticity. Curr Opin Neurobiol 14:318-327.

Diana M, Spiga S, Acquas E (2006) Persistent and reversible morphine withdrawal-induced morphological changes in the nucleus accumbens. Ann N Y Acad Sci 1074:446-457.

Duttaroy A, Yoburn BC (1995) The effect of intrinsic efficacy on opioid tolerance. Anesthesiology 82:1226-1236.

Eisch AJ, Barrot M, Schad CA, Self DW, Nestler EJ (2000) Opiates inhibit neurogenesis in the adult rat hippocampus. Proc Natl Acad Sci U S A 97:7579-7584.

Gáborik Z, Szaszák M, Szidonya L, Balla B, Paku S, Catt KJ, Clark AJ, Hunyady L (2001) Beta-arrestin- and dynamin-dependent endocytosis of the AT1 angiotensin receptor. Mol Pharmacol 59:239-247.

Gaudillière B, Konishi Y, de la Iglesia N, Yao G, Bonni A (2004) A CaMKIINeuroD signaling pathway specifies dendritic morphogenesis. Neuron 41:229-241.

Kayser MS, Nolt MJ, Dalva MB (2008) EphB receptors couple dendritic filopodia motility to synapse formation. Neuron 59:56-69.

Liao D, Lin H, Law PY, Loh HH (2005) Mu-opioid receptors modulate the stability of dendritic spines. Proc Natl Acad Sci U S A 102:1725-1730.

Liao D, Grigoriants OO, Loh HH, Law PY (2007a) Agonist-dependent postsynaptic effects of opioids on miniature excitatory postsynaptic currents in cultured hippocampal neurons. J Neurophysiol 97:1485-1494.

Liao D, Grigoriants OO, Wang W, Wiens K, Loh HH, Law PY (2007b) Distinct effects of individual opioids on the morphology of spines depend upon the internalization of mu opioid receptors. Mol Cell Neurosci 35:456-469.

Lin H, Higgins P, Loh HH, Law PY, Liao D (2009) Bidirectional effects of fentanyl on dendritic spines and AMPA receptors depend upon the internalization of mu opioid receptors. Neuropsychopharmacology 34:2097-2111.

Lou L, Zhou T, Wang P, Pei G (1999) Modulation of Ca2+/calmodulindependent protein kinase II activity by acute and chronic morphine administration in rat hippocampus: differential regulation of alpha and beta isoforms. Mol Pharmacol 55:557-563.

Ma L, Pei G (2007) Beta-arrestin signaling and regulation of transcription. J Cell Sci 120:213-218.

Mei B, Niu L, Cao B, Huang D, Zhou Y (2009) Prenatal morphine exposure alters the layer II/III pyramidal neurons morphology in lateral secondary visual cortex of juvenile rats. Synapse 63:1154-1161.

Pratt KG, Watt AJ, Griffith LC, Nelson SB, Turrigiano GG (2003) Activitydependent remodeling of presynaptic inputs by postsynaptic expression of activated CaMKII. Neuron 39:269-281.

Pu L, Bao GB, Xu NJ, Ma L, Pei G (2002) Hippocampal long-term potentiation is reduced by chronic opiate treatment and can be restored by reexposure to opiates. J Neurosci 22:1914-1921.

Ricalde AA, Hammer RP Jr (1990) Perinatal opiate treatment delays growth of cortical dendrites. Neurosci Lett 115:137-143.

Robinson TE, Gorny G, Savage VR, Kolb B (2002) Widespread but regionally specific effects of experimenter- versus self-administered morphine on dendritic spines in the nucleus accumbens, hippocampus, and neocortex of adult rats. Synapse 46:271-279.

Schafer DA (2004) Regulating actin dynamics at membranes: a focus on dynamin. Traffic 5:463-469.

Seo S, Lim JW, Yellajoshyula D, Chang LW, Kroll KL (2007) Neurogenin and NeuroD direct transcriptional targets and their regulatory enhancers. EMBO J 26:5093-5108.

Sumi M, Kiuchi K, Ishikawa T, Ishii A, Hagiwara M, Nagatsu T, Hidaka H (1991) The newly synthesized selective $\mathrm{Ca} 2+/$ calmodulin dependent protein kinase II inhibitor KN-93 reduces dopamine contents in PC12h cells. Biochem Biophys Res Commun 181:968-975.

Vaillant AR, Zanassi P, Walsh GS, Aumont A, Alonso A, Miller FD (2002) Signaling mechanisms underlying reversible, activity-dependent dendrite formation. Neuron 34:985-998.

Violin JD, Lefkowitz RJ (2007) Beta-arrestin-biased ligands at seventransmembrane receptors. Trends Pharmacol Sci 28:416-422.

von Bohlen und Halbach O (2007) Immunohistological markers for staging neurogenesis in adult hippocampus. Cell Tissue Res 329:409-420.

Wayman GA, Lee YS, Tokumitsu H, Silva A, Soderling TR (2008) Calmodulin-kinases: modulators of neuronal development and plasticity. Neuron 59:914-931.

Zhao H, Loh HH, Law PY (2006) Adenylyl cyclase superactivation induced by long-term treatment with opioid agonist is dependent on receptor localized within lipid rafts and is independent of receptor internalization. Mol Pharmacol 69:1421-1432.

Zheng H, Loh HH, Law PY (2008a) Beta-arrestin-dependent mu-opioid receptor-activated extracellular signal-regulated kinases (ERKs) translocate to nucleus in contrast to $\mathrm{G}$ protein-dependent ERK activation. Mol Pharmacol 73:178-190.

Zheng H, Chu J, Qiu Y, Loh HH, Law PY (2008b) Agonist-selective signaling is determined by the receptor location within the membrane domains. Proc Natl Acad Sci U S A 105:9421-9426.

Zheng H, Loh HH, Law PY (2010a) Agonist-selective signaling of G protein-coupled receptor: mechanisms and implications. IUBMB Life 62:112-119.

Zheng H, Zeng Y, Zhang X, Chu J, Loh HH, Law PY (2010b) Mu-opioid receptor agonists differentially regulate the expression of miR-190 and NeuroD. Mol Pharmacol 77:102-109. 